Proyecciones Journal of Mathematics

Vol. 32, No 3, pp. 245-258, September 2013.

Universidad Católica del Norte

Antofagasta - Chile

\title{
On the instability of a kind of vector functional differential equations of the eighth order with multiple deviating arguments
}

\author{
Cemil Tunç \\ Yüzüncü Yıl University, Turkey \\ Received: August 2012. Accepted : May 2013
}

\begin{abstract}
In this paper, we investigate the instability of solutions to a certain class of nonlinear vector functional differential equations of the eighth order with n-deviating arguments. We employ the LyapunovKrasovskii functional approach and base on the Krasovskii criteria to prove two new theorems on the topic. Our results improve certain results in the literature from scalar functional differential equations to their vectorial forms.
\end{abstract}

Subjclass[2010] : 34K20.

Keywords : Instability, Lyapunov functional, vector functional differential equation, eighth order, multiple deviating arguments. 


\section{Introduction}

The instability analysis of differential equations of the eighth order has received considerable attention in the last two decades. In the literature, the Lyapunov technique has been utilized to study the instability of the solutions of differential equations of the eighth order (Bereketoğlu [2], Iyase [4], Tunç $[6,7,8,9,10]$ and C. Tunç and E. Tunç [11]). Some respective contributions on the topic can be summarized as the following:

First, in 1991, using the Lyapunov technique, Bereketoğlu [2] established certain conditions to the instability of the zero solution of the eighth order scalar differential equation without delay

$$
\begin{aligned}
& x^{(8)}+a_{2} x^{(6)}+a_{3} x^{(5)}+a_{4} x^{(4)}+f_{5}\left(x, x^{\prime}, \ldots, x^{(7)}\right) x^{\prime \prime \prime} \\
& +f_{6}\left(x^{\prime}\right) x^{\prime \prime}+f_{7}\left(x, x^{\prime}, \ldots, x^{(7)}\right) x^{\prime}+f_{8}(x)=0 .
\end{aligned}
$$

Later, in 1996, using the same method, Iyase [4] proved a theorem on the nonexistence of nontrivial periodic solutions to the nonlinear scalar differential equation of the eighth order without delay:

$x^{(8)}+a_{1} x^{(7)}+a_{2} x^{(6)}+a_{3} x^{(5)}+a_{4} x^{(4)}+a_{5} x^{\prime \prime \prime}+f_{6}\left(x^{\prime}\right) x^{\prime \prime}+f_{7}(x) x^{\prime}+f_{8}(x)=0$.

Recently, Tunç $[8,9,10]$ discussed the instability of the zero solution of the eighth order scalar nonlinear differential equations with delay

$$
\begin{aligned}
& x^{(8)}+a_{2} x^{(6)}+a_{3} x^{(5)}+a_{4} x^{(4)}+f_{5}\left(x, x(t-r), x^{\prime}, \ldots, x^{(7)}(t-r)\right) x^{\prime \prime \prime} \\
& +f_{6}\left(x^{\prime}\right) x^{\prime \prime}+f_{7}\left(x, x(t-r), x^{\prime}, \ldots, x^{(7)}(t-r)\right) x^{\prime}+f_{8}(x(t-r))=0, \\
& x^{(8)}+a_{1} x^{(7)}+a_{2} x^{(6)}+a_{3} x^{(5)}+a_{4} x^{(4)}+a_{5} x^{\prime \prime \prime}+f_{6}\left(x^{\prime}\right) x^{\prime \prime} \\
& +f_{7}(x) x^{\prime}+f_{8}\left(x, x(t-r), x^{\prime}, \ldots, x^{(7)}(t-r)\right)=0
\end{aligned}
$$

and

$$
\begin{aligned}
& x^{(8)}+a_{2} x^{(6)}+a_{3} x^{(5)}+a_{4} x^{(4)}+f_{5}\left(x, \ldots, x\left(t-\tau_{n}\right), \ldots, x^{(7)}, \ldots, x^{(7)}\left(t-\tau_{n}\right)\right) x^{\prime \prime \prime} \\
& +f_{6}\left(x^{\prime}\right) x^{\prime \prime}+f_{7}\left(x, \ldots, x\left(t-\tau_{n}\right), \ldots, x^{(7)}, \ldots, x^{(7)}\left(t-\tau_{n}\right)\right) x^{\prime} \\
& +\sum_{i=1}^{n} h_{i}\left(x\left(t-\tau_{i}\right)\right)=0,
\end{aligned}
$$




$$
\begin{aligned}
x^{(8)}+ & a_{1} x^{(7)}+a_{2} x^{(6)}+a_{3} x^{(5)}+a_{4} x^{(4)}+a_{5} x^{\prime \prime \prime}+g_{6}\left(x^{\prime}\right) x^{\prime \prime} \\
& +g_{7}\left(x, \ldots, x\left(t-\tau_{n}\right), x^{\prime}, \ldots, x^{\prime}\left(t-\tau_{n}\right)\right) \\
& +g_{8}\left(x, \ldots, x\left(t-\tau_{n}\right), \ldots, x^{(7)}, \ldots, x^{(7)}\left(t-\tau_{n}\right)\right)=0,
\end{aligned}
$$

respectively.

In this paper, we consider the eighth order nonlinear functional vector differential equations with n-deviating arguments, $\tau_{i},(i=1,2, \ldots, n)$ :

$$
\begin{aligned}
X^{(8)}+ & A_{2} X^{(6)}+A_{3} X^{(5)}+A_{4} X^{(4)} \\
& +F_{5}\left(X, X\left(t-\tau_{1}\right), \ldots, X\left(t-\tau_{n}\right), \ldots, X^{(7)}, \ldots, X^{(7)}\left(t-\tau_{n}\right)\right) X^{\prime \prime \prime} \\
& +F_{6}\left(X^{\prime}\right) X^{\prime \prime} \\
& +F_{7}\left(X, X\left(t-\tau_{1}\right), \ldots, X\left(t-\tau_{n}\right), \ldots, X^{(7)}, \ldots, X^{(7)}\left(t-\tau_{n}\right)\right) X^{\prime} \\
& +\sum_{i=1}^{n} H_{i}\left(X\left(t-\tau_{i}\right)\right)=0
\end{aligned}
$$

and

$$
\begin{aligned}
X^{(8)} & +A_{1} X^{(7)}+A_{2} X^{(6)}+A_{3} X^{(5)}+A_{4} X^{(4)}+A_{5} X^{\prime \prime \prime}+G_{6}\left(X^{\prime}\right) X^{\prime \prime} \\
& +G_{7}\left(X, X\left(t-\tau_{1}\right), \ldots, X\left(t-\tau_{n}\right), \ldots, X^{\prime}, \ldots, X^{\prime}\left(t-\tau_{n}\right)\right) \\
& +G_{8}\left(X, X\left(t-\tau_{1}\right), \ldots, X\left(t-\tau_{n}\right), \ldots, X^{(7)}, \ldots, X^{(7)}\left(t-\tau_{n}\right)\right)=0
\end{aligned}
$$

respectively.

Let $X=X_{1}, X^{\prime}=X_{2}, \ldots, X^{(7)}=X_{8}$. We can write Eq. (1.3) and Eq. (1.4) in the system form 


$$
\begin{aligned}
& X_{i}^{\prime}=X_{i+1},(i=1,2, \ldots, 7) \\
& X_{8}^{\prime}=-A_{2} X_{7}-A_{3} X_{6}-A_{4} X_{5} \\
& -F_{5}\left(X_{1}, \ldots, X_{1}\left(t-\tau_{n}\right), \ldots, X_{8}, \ldots, X_{8}\left(t-\tau_{n}\right)\right) X_{4} \\
& -F_{6}\left(X_{2}\right) X_{3}-F_{7}\left(X_{1}, \ldots, X_{1}\left(t-\tau_{n}\right), \ldots, X_{8}, \ldots, X_{8}\left(t-\tau_{n}\right)\right) X_{2} \\
& -\sum_{i=1}^{n} H_{i}\left(X_{1}\right)+\sum_{i=1}^{n} \int_{t-\tau_{i}}^{t} J_{H_{i}}\left(X_{1}(s)\right) X_{2}(s) d s
\end{aligned}
$$

and

$$
\begin{aligned}
X_{i}^{\prime}= & X_{i+1},(i=1,2, \ldots, 7) \\
X_{8}^{\prime}= & -A_{1} X_{8}-A_{2} X_{7}-A_{3} X_{6}-A_{4} X_{5}-A_{5} X_{4}-G_{6}\left(X_{2}\right) X_{3} \\
& -G_{7}\left(X_{1}, \ldots, X_{1}\left(t-\tau_{n}\right), X_{2}, \ldots, X_{2}\left(t-\tau_{n}\right)\right) \\
& -G_{8}\left(X_{1}, \ldots, X_{1}\left(t-\tau_{n}\right), \ldots, X_{8}, \ldots, X_{8}\left(t-\tau_{n}\right)\right)
\end{aligned}
$$

respectively, where $\tau_{i}$ are certain positive constants, the fixed delays, $t-\tau_{i} \geq 0, A_{1}, \ldots, A_{5}$ are constant $n \times n$ - symmetric matrices, the primes in Eq. (1.3) and Eq. (1.4) denote differentiation with respect to $t, t \in$ $\Re_{+}, \Re_{+}=[0, \infty) ; F_{5}, F_{6}, F_{7}$ and $G_{6}$ are continuous $n \times n-$ symmetric matrix functions for the arguments displayed explicitly, $H_{i}: \Re^{n} \rightarrow \Re^{n}$, $G_{7}: \Re^{2 n(n+1)} \rightarrow \Re^{n}, G_{8}: \Re^{8 n(n+1)} \rightarrow \Re^{n}, G_{7}\left(X_{1}, \ldots, X_{1}\left(t-\tau_{n}\right), 0, X_{2}(t-\right.$ $\left.\left.\tau_{1}\right), \ldots, X_{2}\left(t-\tau_{n}\right)\right)=0, G_{8}\left(0, X_{1}\left(t-\tau_{1}\right), \ldots, X_{8}\left(t-\tau_{n}\right)\right)=0$ and $H_{i}(0)=0$, $H_{i}, G_{7}$ and $G_{8}$ are continuous for all of their respective arguments. The Jacobian matrices of $H_{i}(X)$ are given by

$$
J_{H_{1}}(X)=\left(\frac{\partial h_{1 i}}{\partial x_{j}}\right), \ldots, J_{H_{n}}(X)=\left(\frac{\partial h_{n i}}{\partial x_{j}}\right)(i, j=1,2, \ldots, n),
$$

where $\left(x_{1}, \ldots, x_{n}\right)$ and $\left(h_{1 i}\right), \ldots,\left(h_{n i}\right)$ are the components of $X$ and $H_{i}$, respectively. It is also assumed that the Jacobian matrices $J_{H_{i}}(X)$ exist and are continuous. The existence and uniqueness of the solutions of Eq. (1.3) and Eq. (1.4) are assumed (see È l'sgol'ts ([3], pp.14, 15). Throughout what follows $X_{1}(t), \ldots, X_{8}(t)$ are abbreviated as $X_{1}, \ldots, X_{8}$, respectively. 
Consider the linear constant coefficient differential equation of the eighth order

$$
x^{(8)}+a_{1} x^{(7)}+a_{2} x^{(6)}+a_{3} x^{(5)}+a_{4} x^{(4)}+a_{5} x^{\prime \prime \prime}+a_{6} x^{\prime \prime}+a_{7} x^{\prime}+a_{8} x=0
$$

and its auxiliary equation

$$
\psi(\lambda) \equiv \lambda^{8}+a_{1} \lambda^{7}+\ldots+a_{7} \lambda+a_{8}=0 .
$$

If $\beta$ is an arbitrary real number, then the real part of $\psi(i \beta)$ is given by $\phi(\beta)=\beta^{8}-a_{2} \beta^{6}+a_{4} \beta^{4}-a_{6} \beta^{2}+a_{8}$.

It is also known that if $a_{2} \leq 0, a_{4} \geq 0, a_{6} \leq 0, a_{8}>0$

in which case $\phi(\beta)>0$, then the auxiliary equation cannot have any purely imaginary root whatever. It therefore follows from general theory that Eq. (1.7) does not has a periodic solution except $x=0$. An analogous consideration of the imaginary part of $\psi(i \beta)$ also leads to conditions on $a_{1}$, $a_{3}, a_{5}$ and $a_{7}$ for the nonexistence of any periodic solution of Eq. (1.7) other than $x=0$.
Besides, if $\mathrm{a}_{1}=0$
$a_{8} \neq 0$,

then the sum of the roots of (1.8) equals zero and each of them is different from zero, respectively. Furthermore, a necessary and sufficient condition for $(1.8)$, with $a_{1}=0$, to have a purely imaginary $\operatorname{root} \lambda=i \beta$ ( $\beta$ real) is that the following two equations

$$
a_{3} \beta^{4}-a_{5} \beta^{2}+a_{7}=0
$$

and

$$
\beta^{8}-a_{2} \beta^{6}+a_{4} \beta^{4}-a_{6} \beta^{2}+a_{8}=0
$$

are simultaneously satisfied. If $a_{3} \neq 0$, the left hand side of Eq. (1.9) can be rewritten in the form

$$
a_{3}\left(\beta^{2}-\frac{a_{5}}{2 a_{3}}\right)^{2}+a_{7}-\frac{a_{5}^{2}}{4 a_{3}} \text {. }
$$

Therefore, if

$$
a_{3} \neq 0,\left(a_{7}-\frac{a_{5}^{2}}{4 a_{3}}\right) \operatorname{sgna}_{3}>0,
$$

then the estimate (1.9) cannot be satisfied, and Eq. (1.8) would not have any purely imaginary root whatever. Hence, Eq. (1.8) has at the least one 
root $\lambda_{0}=\alpha_{0}+i \beta_{0},\left(\alpha_{0}, \beta_{0}\right.$ real $)$ with $\alpha_{0}>0$ provided that $a_{1}=0, a_{8} \neq 0$ and (1.10) hold.

The aim of this paper is to give the extensions of these results to Eq. (1.3) and Eq. (1.4). We extend the results obtained in the scalar cases for Eq. (1.1) and Eq. (1.2) to vector delay differential equations, Eq. (1.3) and Eq. (1.4).

Let $r \geq 0$ be given, and let $C=C\left([-r, 0], \Re^{n}\right)$ with

$$
\|\phi\|=\max _{-r \leq s \leq 0}|\phi(s)|, \quad \phi \in C .
$$

For $H>0$ define $C_{H} \subset C$ by

$$
C_{H}=\{\phi \in C:\|\phi\|<H\} .
$$

If $x:[-r, A) \rightarrow \Re^{n}$ is continuous, $0<A \leq \infty$, then, for each $t$ in $[0, A)$, $x_{t}$ in $C$ is defined by

$$
x_{t}(s)=x(t+s),-r \leq s \leq 0, t \geq 0 .
$$

Let $G$ be an open subset of $C$ and consider the general autonomous differential system with finite delay

$$
\dot{x}=F\left(x_{t}\right), F(0)=0, x_{t}=x(t+\theta),-r \leq \theta \leq 0, t \geq 0,
$$

where $F: G \rightarrow \Re^{n}$ is continuous and maps closed and bounded sets into bounded sets. It follows from these conditions on $F$ that each initial value problem

$$
\dot{x}=F\left(x_{t}\right), x_{0}=\phi \in G
$$

has a unique solution defined on some interval $[0, A), 0<A \leq \infty$. This solution will be denoted by $x(\phi)($.$) so that x_{0}(\phi)=\phi$.

Definition 1.1. The zero solution, $x=0$, of $\dot{x}=F\left(x_{t}\right)$ is stable if for each $\varepsilon>0$ there exists $\delta=\delta(\varepsilon)>0$ such that $\|\phi\|<\delta$ implies that $|x(\phi)(t)|<\varepsilon$ for all $t \geq 0$. The zero solution is said to be unstable if it is not stable. 


\section{Main results}

We need the following lemma.

Lemma 2.1. (Bellman [1]) Let $A$ be a real symmetric $n \times n-$ matrix and

$$
a^{\prime} \geq \lambda_{i}(A) \geq a>0,(i=1,2, \ldots, n),
$$

where $a^{\prime}$ and $a$ are constants, and $\lambda_{i}(A)$ are the eigenvalues of the matrix A. Then

$$
a^{\prime}\langle X, X\rangle \geq\langle A X, X\rangle \geq a\langle X, X\rangle
$$

and

$$
a^{\prime^{2}}\langle X, X\rangle \geq\langle A X, A X\rangle \geq a^{2}\langle X, X\rangle
$$

The first main result is the following theorem.

Let $\tau=\max \tau_{i}, \quad(i=1,2, \ldots, n)$.

Theorem 2.1. Let all the assumptions imposed to $A_{2}, A_{3}, A_{4}, F_{5}, F_{6}, F_{7}$ and $H_{i}$ and

$\left.H_{i}(0)=0, H_{i}\left(X_{1}\right) \neq 0,\left(X_{1} \neq 0\right),\left|\lambda_{i}\left(J_{H_{i}}\left(X_{1}\right)\right)\right| \leq \delta_{i}, \delta_{i}>0, \lambda_{i}\left(A_{3}().\right)\right) \geq a_{3}$

and

$$
\lambda_{i}\left(F_{7}\left(X_{1}, \ldots, X_{8}\left(t-\tau_{n}\right)\right)\right) \operatorname{sgna}_{3}-\frac{1}{4\left|a_{3}\right|} \lambda_{i}\left(F_{5}\left(X_{1}, \ldots, X_{8}\left(t-\tau_{n}\right)\right)\right)^{2} \geq \delta>0
$$

hold for arbitrary $X_{1}, \ldots, X_{8}\left(t-\tau_{n}\right)$, where $a_{3}(\neq 0), \delta_{i}$ and $\delta$ are constants.

If $\tau<\frac{\delta}{\sqrt{n}\left(\delta_{1}+\ldots+\delta_{n}\right)}$, then the zero solution of Eq. (1.3) is unstable.

Proof. Let the function $V()=.V\left(X_{1}, \ldots, X_{8}\right)$ be defined by

$$
\begin{aligned}
V(.)= & -\left\langle X_{2}, X_{8}\right\rangle-\left\langle A_{2} X_{2}, X_{6}\right\rangle-\left\langle A_{3} X_{2}, X_{5}\right\rangle-\left\langle A_{4} X_{2}, X_{4}\right\rangle \\
& -\int_{0}^{1}\left\langle H_{1}(\sigma X), X\right\rangle d \sigma-\ldots-\int_{0}^{1}\left\langle H_{n}(\sigma X), X\right\rangle d \sigma \\
& +\left\langle X_{3}, X_{7}\right\rangle+\left\langle A_{2} X_{3}, X_{5}\right\rangle+\left\langle A_{3} X_{3}, X_{4}\right\rangle-\left\langle X_{4}, X_{6}\right\rangle \\
& +\frac{1}{2}\left\langle A_{4} X_{3}, X_{3}\right\rangle-\frac{1}{2}\left\langle A_{2} X_{4}, X_{4}\right\rangle+\frac{1}{2}\left\langle X_{5}, X_{5}\right\rangle \\
& -\int_{0}^{1}\left\langle F_{6}(2) X_{2}, X_{2}\right\rangle d \sigma .
\end{aligned}
$$


We define a Lyapunov functional $V_{1}(.) \equiv V_{1}\left(X_{1 t}, \ldots, X_{8 t}\right)$ by

$$
V_{1}(.)=V \operatorname{sgna}_{3}-\sum_{i=1}^{n} \lambda_{i} \int_{-\tau_{i}}^{0} \int_{t+s}^{t}\left\|X_{2}(\theta)\right\|^{2} d \theta d s,
$$

where $s$ is a real variable such that the integrals $\int_{-\tau_{i}}^{0} \int_{t+s}^{t}\left\|X_{2}(\theta)\right\|^{2} d \theta d s$ are non-negative, and $\lambda_{i}$ are certain positive constants to be determined later in the proof.

It follows that

$$
V_{1}(0,0,0,0,0,0,0,0)=0 .
$$

Let

$$
\bar{\varepsilon}=\left(0,0,\left(\varepsilon_{31}, \ldots, \varepsilon_{3 n}\right) \operatorname{sgna}_{3}, 0,0,0,\left(1+\left|a_{4}\right|\right)\left(\varepsilon_{71}, \ldots, \varepsilon_{7 n}\right), 0\right) .
$$

$$
\begin{gathered}
\text { Hence } \quad \mathrm{V}_{1}\left(0,0,\left(\varepsilon_{31}, \ldots, \varepsilon_{3 n}\right) \operatorname{sgna}_{3}, 0,0,0,\left(1+\left|a_{4}\right|\right)\left(\varepsilon_{71}, \ldots, \varepsilon_{7 n}\right), 0\right) \\
\geq\left(1+\left|a_{4}\right|+\frac{1}{2} a_{4} \operatorname{sgna}_{3}\right)\|\bar{\varepsilon}\|^{2}>0
\end{gathered}
$$

for all $\bar{\varepsilon}(\neq 0)$ so that every neighborhood of the origin in the $\left(X_{1}, \ldots, X_{8}\right)-$ space contains the points $\left(\xi_{1}, \ldots, \xi_{8}\right)$ such that $V_{1}\left(\xi_{1}, \ldots, \xi_{8}\right)>0$.

Let ...

$$
\left(X_{1}, \ldots, X_{8}\right)=\left(X_{1}(t), \ldots, X_{8}(t)\right)
$$

be an arbitrary solution of (1.5).

The time derivative of the Lyapunov functional $V_{1}($.$) with respect to$ (1.5) results in 


$$
\begin{aligned}
\dot{V}_{1}(.)= & \left\{\left\langle A_{3} X_{4}, X_{4}\right\rangle+\left\langle F_{5}(.) X_{2}, X_{4}\right\rangle+\left\langle F_{7}(.) X_{2}, X_{2}\right\rangle\right\} \operatorname{sgna}_{3} \\
& \left.-<\operatorname{sgna}_{3} X_{2}, \int_{t-\tau_{1}}^{t} J_{H_{1}}\left(X_{1}(s)\right) X_{2}(s) d s\right\rangle \\
& \left.-\ldots-<\operatorname{sgna}_{3} X_{2}, \int_{t-\tau_{n}}^{t} J_{H_{n}}\left(X_{1}(s)\right) X_{2}(s) d s\right\rangle \\
& -\left\langle\lambda_{1} \tau_{1} X_{2}, X_{2}\right\rangle-\ldots-\left\langle\lambda_{n} \tau_{n} X_{2}, X_{2}\right\rangle \\
& +\lambda_{1} \int_{t-\tau_{1}}^{t}\left\|X_{2}(\theta)\right\|^{2} d \theta+\ldots+\lambda_{n} \int_{t-\tau_{n}}^{t}\left\|X_{2}(\theta)\right\|^{2} d \theta \\
& \geq\left|a_{3}\right|\left\|X_{4}+2^{-1}\left|a_{3}^{-1}\right| F_{5}(.) X_{2} \operatorname{sgna}\right\|^{2} \\
& +\left\langle\operatorname{sgna} \|_{3} F_{7}(.) X_{2}, X_{2}\right\rangle-<4^{-1}\left|a_{3}^{-1}\right| F_{5}(.) X_{2}, F_{5}(.) X_{2}> \\
& -<\operatorname{sgna}_{3} X_{2}, \int_{t-\tau_{1}}^{t} J_{H_{1}}\left(X_{1}(s)\right) X_{2}(s) d s> \\
& -\ldots-<\operatorname{sgna}_{3} X_{2}, \int_{t-\tau_{n}}^{t} J_{H_{n}}\left(X_{1}(s)\right) X_{2}(s) d s> \\
& -\left\langle\lambda_{1} \tau_{1} X_{2}, X_{2}\right\rangle-\left\langle\lambda_{2} \tau_{2} X_{2}, X_{2}\right\rangle-\ldots-\left\langle\lambda_{n} \tau_{n} X_{2}, X_{2}\right\rangle \\
& +\lambda_{1} \int_{t-\tau_{1}}^{t}\left\|X_{2}(\theta)\right\|^{2} d \theta+\ldots+\lambda_{n} \int_{t-\tau_{n}}^{t}\left\|X_{2}(\theta)\right\|^{2} d \theta .
\end{aligned}
$$

Using the assumptions of Theorem 2.1 and the Schwartz inequality, we have

$$
\begin{gathered}
-<\operatorname{sgna}_{3} X_{2}, \int_{t-\tau_{1}}^{t} J_{H_{1}}\left(X_{1}(s)\right) X_{2}(s) d s> \\
\geq-\left\|X_{2}\right\|\left\|\int_{t-\tau_{1}}^{t} J_{H_{1}}\left(X_{1}(s)\right) X_{2}(s) d s\right\| \\
\geq-\sqrt{n} \delta_{1}\left\|X_{2}\right\|\left\|\int_{t-\tau_{1}}^{t} X_{2}(s)\right\| d s \\
\geq-\sqrt{n} \delta_{1}\left\|X_{2}\right\| \int_{t-\tau_{1}}^{t}\left\|X_{2}(s)\right\| d s \\
\geq-\frac{1}{2} \sqrt{n} \delta_{1} \tau_{1}\left\|X_{2}\right\|^{2}-\frac{1}{2} \sqrt{n} \delta_{1} \int_{t-\tau_{1}}^{t}\left\|X_{2}(s)\right\|^{2} d s
\end{gathered}
$$




$$
\begin{aligned}
& -<\operatorname{sgna}_{3} X_{2}, \int_{t-\tau_{2}}^{t} J_{H_{2}}\left(X_{1}(s)\right) X_{2}(s) d s> \\
& \geq-\left\|X_{2}\right\|\left\|\int_{t-\tau_{2}}^{t} J_{H_{2}}\left(X_{1}(s)\right) X_{2}(s) d s\right\| \\
& \geq-\sqrt{n} \delta_{2}\left\|X_{2}\right\|\left\|\int_{t-\tau_{2}}^{t} X_{2}(s)\right\| d s \\
& \geq-\sqrt{n} \delta_{2}\left\|X_{2}\right\| \int_{t-\tau_{2}}^{t}\left\|X_{2}(s)\right\| d s \\
& \geq-\frac{1}{2} \sqrt{n} \delta_{2} \tau_{2}\left\|X_{2}\right\|^{2}-\frac{1}{2} \sqrt{n} \delta_{2} \int_{t-\tau_{2}}^{t}\left\|X_{2}(s)\right\|^{2} d s, \ldots, \\
& -<s g n a_{3} X_{2}, \int_{t-\tau_{n}}^{t} J_{H_{n}}\left(X_{1}(s)\right) X_{2}(s) d s> \\
& \geq-\left\|X_{2}\right\|\left\|\int_{t-\tau_{n}}^{t} J_{H_{n}}\left(X_{1}(s)\right) X_{2}(s) d s\right\| \\
& \geq-\sqrt{n} \delta_{n}\left\|X_{2}\right\| \int_{t-\tau_{n}}^{t} X_{2}(s) \| d s \\
& \geq-\sqrt{n} \delta_{n}\left\|X_{2}\right\| \int_{t-\tau_{n}}^{t}\left\|X_{2}(s)\right\| d s \\
& \geq-\frac{1}{2} \sqrt{n} \delta_{n} \tau_{n}\left\|X_{2}\right\|^{2}-\frac{1}{2} \sqrt{n} \delta_{n} \int_{t-\tau_{n}}^{t}\left\|X_{2}(s)\right\|^{2} d s
\end{aligned}
$$

so that

$$
\begin{aligned}
\dot{V}_{1}(.) \geq & \left|a_{3}\right|\left\|X_{4}+2^{-1}\left|a_{3}^{-1}\right| F_{5}(.) X_{2} \operatorname{sgna}_{3}\right\|^{2} \\
& +\left\langle\operatorname{sgna}_{3} F_{7}(.) X_{2}, X_{2}\right\rangle-<4^{-1}\left|a_{3}^{-1}\right| F_{5}(.) X_{2}, F_{5}(.) X_{2}> \\
& -\left(\lambda_{1}+\frac{1}{2} \sqrt{n} \delta_{1}\right) \tau_{1}\left\|X_{2}\right\|^{2}-\ldots-\left(\lambda_{n}+\frac{1}{2} \sqrt{n} \delta_{n}\right) \tau_{n}\left\|X_{2}\right\|^{2} \\
& +\left(\lambda_{1}-\frac{1}{2} \sqrt{n} \delta_{1}\right) \int_{t-\tau_{1}}^{t}\left\|X_{2}(s)\right\|^{2} d s+\ldots+\left(\lambda_{n}-\frac{1}{2} \sqrt{n} \delta_{n}\right) \\
& \quad \int_{t-\tau_{n}}^{t}\left\|X_{2}(s)\right\|^{2} d s .
\end{aligned}
$$

Let $\lambda_{i}=\frac{1}{2} \sqrt{n} \delta_{i}$ and $\tau=\max \tau_{i}, \quad(i=1,2, \ldots, n)$. Hence, we obtain

$$
\begin{aligned}
\dot{V}_{1}(.) \geq & \left\langle\operatorname{sgna}_{3} F_{7}(.) X_{2}, X_{2}\right\rangle-<4^{-1}\left|a_{3}^{-1}\right| F_{5}(.) X_{2}, F_{5}(.) X_{2}> \\
& -\sqrt{n} \delta_{1} \tau_{1}\left\|X_{2}\right\|^{2}-\ldots-\sqrt{n} \delta_{n} \tau_{n}\left\|X_{2}\right\|^{2} \\
& \geq\left\{\delta-\sqrt{n}\left(\delta_{1}+\ldots+\delta_{n}\right) \tau\right\}\left\|X_{2}\right\|^{2} .
\end{aligned}
$$


If $\tau<\frac{\delta}{\sqrt{n}\left(\delta_{1}+\ldots+\delta_{n}\right)}$, then, for some positive constant $k$, we have

$$
\dot{V}_{1}(.) \geq k\left\|X_{2}\right\|^{2}>0 \text {. }
$$

Finally, $\dot{V}_{1}()=$.0 for all $t \geq 0$ necessarily implies that $X_{2}=0$. Hence, it follows that

$$
X_{1}=\xi_{1}(\text { constant vector }), \quad X_{2}=X^{\prime}=0, \ldots, X_{8}=X^{(7)}=0
$$

for all $t \geq 0$ so that

$$
\geq-\left\|X_{2}\right\|\left\|\int_{t-\tau_{1}}^{t} J_{H_{1}}\left(X_{1}(s)\right) X_{2}(s) d s\right\|
$$

Therefore, the estimates $\dot{V}_{1}()=$.0 and (1.5) imply $X_{1}=X_{2}=\ldots=$ $X_{8}=0$, since $H_{i}\left(\xi_{1}\right)=0$ if and only if $\xi_{1}=0$. It now follows that functional $V_{1}$ thus has all the requisite Krasovskii [5] properties subject to the conditions of Theorem 2.1. By this discussion, we can conclude that the zero solution of Eq. (1.3) is unstable.

The proof of Theorem 2.1 is completed.

The second main result of this paper is given by the following theorem.

Theorem 2.2. Let all the assumptions imposed to $A_{1}, \ldots, A_{5}, G_{6}, G_{7}$ and $G_{8}$ and the conditions

$$
\begin{aligned}
& \lambda_{i}\left(A_{1}\right) \geq a_{1}>0, \lambda_{i}\left(A_{2}\right) \leq a_{2}<0, \lambda_{i}\left(A_{4}\right) \geq a_{4}>0 \\
& \lambda_{i}\left(G_{6}\left(X_{2}\right)\right) \leq 0,\left\langle G_{7}\left(X_{1}, \ldots, X_{2}\left(t-\tau_{n}\right)\right), X_{1}\right\rangle \geq 0
\end{aligned}
$$

and

$$
\left\langle G_{8}\left(X_{1}, \ldots, X_{8}\left(t-\tau_{n}\right)\right), X_{1}\right\rangle>0,\left(X_{1} \neq 0\right),
$$

hold for arbitrary $X_{1}, X_{1}\left(t-\tau_{1}\right), \ldots, X_{8}\left(t-\tau_{n}\right)$, where $a_{1}, a_{2}$ and $a_{4}$ are certain constants.

Then, the zero solution of Eq. (1.4) is unstable. 
Proof. Let the Lyapunov function $V_{2}()=.V_{2}\left(X_{1}, \ldots, X_{8}\right)$ be defined by

$$
\begin{aligned}
V_{2}(.)= & -\left\langle X_{1}, X_{8}\right\rangle-\left\langle A_{1} X_{1}, X_{7}\right\rangle-\left\langle A_{2} X_{1}, X_{6}\right\rangle-\left\langle A_{3} X_{1}, X_{5}\right\rangle \\
& -\left\langle A_{4} X_{1}, X_{4}\right\rangle-\left\langle A_{5} X_{1}, X_{3}\right\rangle+\left\langle X_{2}, X_{7}\right\rangle+\left\langle A_{1} X_{2}, X_{6}\right\rangle \\
& +\left\langle A_{2} X_{2}, X_{5}\right\rangle+\left\langle A_{3} X_{2}, X_{4}\right\rangle+\left\langle A_{4} X_{2}, X_{3}\right\rangle+\frac{1}{2}\left\langle A_{5} X_{2}, X_{2}\right\rangle \\
& -\left\langle X_{3}, X_{6}\right\rangle-\left\langle A_{1} X_{3}, X_{5}\right\rangle-\left\langle A_{2} X_{3}, X_{4}\right\rangle-\frac{1}{2}\left\langle A_{3} X_{3}, X_{3}\right\rangle \\
& \left.+\left\langle X_{4}, X_{5}\right\rangle+\frac{1}{2}\left\langle A_{1} X_{4}, X_{4}\right\rangle-<X_{1}, \int_{0}^{1} G_{6}\left(\sigma X_{2}\right) X_{2} d \sigma\right\rangle .
\end{aligned}
$$

It follows that

$$
V_{2}(0,0,0,0,0,0,0,0)=0 \text {. }
$$

Let

$$
\varepsilon *=\left(\varepsilon_{41}, \ldots, \varepsilon_{4 n}\right) .
$$

Then

$$
V_{2}(0,0,0, \varepsilon *, 0,0,0,0)=\frac{1}{2} a_{1}\|\varepsilon *\|^{2}>0
$$

for $\varepsilon *(\neq 0)$ so that every neighborhood of the origin in the $\left(X_{1}, X_{2}, \ldots, X_{8}\right)-$ space contains points $\left(\rho_{1}, \rho_{2}, \ldots, \rho_{8}\right)$ such that $V_{2}\left(\rho_{1}, \rho_{2}, \ldots, \rho_{8}\right)>0$.

Let

$$
\left(X_{1}, \ldots, X_{8}\right)=\left(X_{1}(t), \ldots, X_{8}(t)\right)
$$

be an arbitrary solution of (1.6).

By an elementary calculation along the solutions of (1.6), the time derivative of the function $V_{2}$ results in

$$
\begin{aligned}
\dot{V}_{2}(.)= & \left\langle X_{5}, X_{5}\right\rangle-\left\langle A_{2} X_{4}, X_{4}\right\rangle+\left\langle A_{4} X_{3}, X_{3}\right\rangle \\
& +\left\langle G_{7}\left(X_{1}, \ldots, X_{8}\left(t-\tau_{n}\right)\right), X_{1}\right\rangle \\
& +\left\langle G_{8}\left(X_{1}, \ldots, X_{8}\left(t-\tau_{n}\right)\right), X_{1}\right\rangle \\
& -<X_{2}, \int_{0}^{1} G_{6}\left(\sigma X_{2}\right) X_{2} d \sigma>.
\end{aligned}
$$

Using the assumptions of Theorem 2.2, $\left.\lambda_{i}\left(A_{2}\right) \leq a_{2}<0, \lambda_{i}\left(A_{4}\right) \geq a_{4}\right\rangle$ $0, \lambda_{i}\left(G_{6}\left(x_{2}\right)\right) \leq 0, X_{1} G_{7}() \geq$.0 and $X_{1} G_{8}()>0,.\left(X_{1} \neq 0\right)$, it follows that

$$
\begin{aligned}
\dot{V}_{2}(.) \geq & \left\|X_{5}\right\|^{2}-a_{2}\left\|X_{4}\right\|^{2}+a_{4}\left\|X_{3}\right\|^{2} \\
& +\left\langle G_{7}\left(X_{1}, \ldots, X_{8}(t-r)\right), X_{1}\right\rangle> \\
& +\left\langle G_{8}\left(X_{1}, \ldots, X_{8}(t-r)\right), X_{1}\right\rangle \\
& -<X_{2}, \int_{0}^{1} G_{6}\left(\sigma X_{2}\right) X_{2} d \sigma>0 .
\end{aligned}
$$

Thus, if the assumptions of Theorem 2.2 hold, then $\dot{V}_{2}($.$) is positive$ semi definite. 
On the other hand, $\dot{V}_{2}=0$ for all $t \geq 0$ necessarily implies that $X_{1}=0$ and therefore also that

$$
X_{1}=X=0, X_{2}=X^{\prime}=0, \ldots, X_{8}=X^{(7)}=0
$$

for all $t \geq 0$ so that

$$
X_{1}=X_{2}=\ldots=X_{8}=0,(t \geq 0)
$$

On the other hand, noting $\dot{V}_{2}()=$.0 and (1.6), we obtain $X_{1}=X_{2}=$ $\ldots=X_{8}=0$ since $G_{8}\left(\rho_{1}, \ldots, \rho_{8}\left(t-\tau_{n}\right)\right)=0$ and $G_{7}\left(\rho_{1}, \ldots, \rho_{2}\left(t-\tau_{n}\right)\right)=0$, if and only if $\rho_{1}=\rho_{2}=0$. It now follows that the Lyapunov function $V_{2}($.$) thus satisfies all the requisite Krasovskii [5] properties subject to the$ conditions of Theorem 2. By the above discussion, we conclude that the zero solution of Eq. (1.6) is unstable.

The proof of Theorem 2 is completed.

\section{References}

[1] R. Bellman, Introduction to matrix analysis. Reprint of the second (1970) edition. With a foreword by Gene Golub. Classics in Applied Mathematics, 19. Society for Industrial and Applied Mathematics (SIAM), Philadelphia, PA, (1997).

[2] H. Bereketoğlu, On the instability of trivial solutions of a class of eighth-order differential equations, Indian J. Pure Appl. Math., 22, no. 3, pp. 199-202, (1991).

[3] L. È. Èl'sgol'ts, Introduction to the theory of differential equations with deviating arguments. Translated from the Russian by Robert J. McLaughlin Holden-Day Inc., San Francisco, Calif.-LondonAmsterdam, (1966).

[4] S. A. Iyase, Periodic solutions of certain eighth order differential equations, J. Nigerian Math. Soc., 14/15 (1995/96), 31-39.

[5] N. N. Krasovskii, On conditions of inversion of A. M. Lyapunov's theorems on instability for stationary systems of differential equations, (Russian) Dokl. Akad. Nauk SSSR (N.S.) 101, pp. 17-20, (1955). 
[6] C. Tunç, Instability of solutions of a certain non-autonomous vector differential equation of eighth-order, Ann. Differential Equations 22, no. 1 , pp. $7-12,(2006)$.

[7] C. Tunç, Nonexistence of nontrivial periodic solutions to a class of nonlinear differential equations of eighth order, Bull. Malays. Math. Sci. Soc., (2) 32, no. 3, pp. 307-311, (2009).

[8] C. Tunç, On the instability of solutions of an eighth order nonlinear differential equation of retarded type, Proyecciones 30, no. 1, pp. 43 $50,(2011)$.

[9] C. Tunç, An instability theorem for solutions of a kind of eighth order nonlinear delay differential equations, World Applied Sciences Journal 12 (5), pp. 619-623, (2011).

[10] C. Tunç, Instability of solutions for nonlinear differential equations of eighth order with multiple deviating arguments, J. Appl. Math. Inform., 31, no. 4-5, (2012).

[11] C. Tunç and E. Tunç, An instability theorem for a class of eighth-order differential equations, (Russian) Differ. Uravn., 42, no. 1, 135-138, 143; translation in Differ. Equ. 42, no. 1, pp. 150-154; (2006).

\section{Cemil Tunç}

Department of Mathematics, Faculty of Sciences, Yüzüncü Yıl University, 65080, van-Turkey

e-mail : cemtunc@yahoo.com 